\title{
Histone Deacetylase 6 Regulates the Activation of MI Macrophages by the Glycolytic Pathway During Acute Liver Failure
}

This article was published in the following Dove Press journal: Journal of Inflammation Research

\section{Yao Wang* \\ Xun $\mathrm{Li}^{*}$ \\ Qian Chen (D) \\ Fangzhou Jiao \\ Chunxia Shi \\ Maohua Pei \\ Luwen Wang \\ Zuojiong Gong (D)}

Department of Infectious Diseases, Renmin Hospital of Wuhan University, Wuhan, 430060, People's Republic of China

*These authors contributed equally to this work
Correspondence: Zuojiong Gong;

Luwen Wang

Department of Infectious Diseases,

Renmin Hospital of Wuhan University,

238 Jiefang Road, Wuhan, 430060,

People's Republic of China

Email zjgong@163.com;

wangluw8253@163.com
Background: The glycolysis pathway of M1 macrophages is a key factor affecting the inflammatory response. The aim of this article is to investigate the role of histone deacetylase 6 (HDAC6) in the M1 macrophage glycolysis pathway during acute liver failure (ALF).

Methodology: Targeted metabolomics for quantitative analysis of energy metabolites technology was used to detect the characteristics of energy metabolism for 8 ALF patients and 8 normal volunteers. The ALF mice model was intervened with HDAC6 inhibitor ACY1215. iTRAQ/TMT quantitative proteomics was used to detect protein expression in livers in different mice groups. The liver function, energy metabolites, M1 macrophages, cytokines, and pathological structure, DDX3X, NLRP3 and DNMT1 in liver tissue were detected. The changes of the above molecules were verified in cell groups.

Results: ALF patients and mice have significant energy metabolism disorders, accompanied by activation of M1 macrophages. After the intervention of ACY-1215, the activated M1 macrophages and cytokines levels in the mouse liver were reduced. The levels of IDH1, MDH1, and ATP were significantly increased. The expression of DDX3X increased, while the expression of NLRP3 and DNMT1 decreased. ACY-1215 could reduce the model cell apoptosis level and inflammatory response, and improve energy metabolism. It could also promote the expression of DDX3X, and inhibit the expression of NLRP3 and DNMT1.

Conclusion: ACY-1215 could inhibit the activation of M1 macrophages by improving the glycolytic pathway through regulating DNMT1 and DDX3X/NLRP3 signals to alleviate ALF.

Keywords: HDAC6, M1 macrophage, metabolic reprogramming, acute liver failure

\section{Introduction}

Acute liver failure (ALF) refers to the clinical syndrome with large-scale necrosis of hepatocytes or severe liver function damage. The clinical manifestations are mainly jaundice, ascites, hepatic encephalopathy, and coagulopathy. ${ }^{1}$ Although a comprehensive set of treatment methods have been concluded, no breakthrough has been achieved so far. ${ }^{2}$ It is reported that intestinal flora disturbances and intestinal endotoxemia (ITEM) plays an important role in the pathogenesis of $\mathrm{ALF}^{3}$ Once the intestinal mucosal barrier of ALF patients is damaged, harmful substances such as bacteria and endotoxins in the intestinal cavity would pass through the intestinal mucosa and enter blood circulation in the body. ${ }^{4}$

Endotoxin exists in the cell wall of Gram-negative bacteria and its main component is lipopolysaccharide (LPS). The endotoxins that enter the liver could 
induce localized non-specific hypersensitivity reactions, and could cause hepatic microcirculation disorders and severe hepatocytes damage. ${ }^{5}$ During the acute inflammatory response phase of ALF, LPS can stimulate the production of classically activated macrophages (named M1 type macrophages). ${ }^{6}$ Activated M1 macrophages show a strong pro-inflammatory effect. They could further release a large number of inflammatory mediators, such as tumor necrosis factor alpha (TNF- $\alpha$ ), interleukin (IL)-6, IL-1 $\beta$, and promote liver damage. ${ }^{7}$ Studies have shown that the activation of M1 macrophages is accompanied by disorder of energy metabolism. The activation of M1 macrophages requires energy to be obtained through the glycolysis pathway during tricarboxylic acid cycle (TCA) in mitochondria. ${ }^{8}$ During the process, the TCA cycle will be broken. The aggregated metabolites, such as succinate, can further promote the aggregation of hypoxia-inducible factor $1 \alpha$ (HIF $1 \alpha)$ and induce the production of the inflammatory factor IL-1 $\beta .^{9}$ Therefore, if the mitochondrial glycolysis pathway of M1 macrophages is reprogrammed, the acute inflammatory response would be alleviated.

Histone acetylation is regulated by histone acetyltransferase (HAT) and histone deacetylase (HDAC). ${ }^{10}$ Under normal physiological circumstances, the structure of HAT and HDAC proteins and enzyme activity maintain a high balance, called "acetylation dynamic equilibrium", which plays an important role in maintaining homeostasis in cells. $^{11}$ In our previous studies, the HDAC6 inhibitor ACY-1215 can inhibit hepatocyte necrosis by promoting autophagy, reducing apoptosis, regulating mitochondrialmediated oxidative stress, inhibiting the TLR4-MAPK/ $\mathrm{NF}-\kappa \mathrm{B}$ signaling pathway in ALF mice or the LPSinduced inflammatory response in RAW264.7 cells. ${ }^{12-14}$ More importantly, nucleotide-binding domain, leucine-rich repeat containing receptor (NLR) family pyrin domain containing 3 (NLRP3) inflammasome and stress granules (SGs) complex competitively bind DEAD (Asp-Glu-AlaAsp) box polypeptide $3 \mathrm{X}$-linked (DDX3X). DDX3X, as an important component of stress granules, also interacts with NLRP3 in bone marrow-derived macrophages stimulated by LPS. DDX3X induces inflammasome activation when it binds to NLRP3 under cellular stress. ${ }^{15}$ DDX $3 \mathrm{X} /$ NLRP3 is a key regulatory pathway of cell survival and pyroptosis, and it might be an important target in the process of inflammation activation. ${ }^{15}$ Therefore, whether the DDX3X/NLRP3 signaling pathway can participate in the glycolysis of cells during the activation of M1 macrophages in ALF, and whether ACY-1215 can promote the energy metabolism of M1 macrophages by regulating the DDX3X/NLRP3 pathway are worth further studies.

This project intends to establish a cellular and mice ALF model. The intervention of HDAC6 inhibitor ACY1215 would be used to clarify the effect of HDAC6 on the glycolytic pathway of M1 macrophages during ALF.

\section{Materials and Methods}

\section{Serum Collection and Energy Metabolite Examination}

All investigations with human subjects were conducted according to the principles expressed in the Helsinki Declaration. The study received the signed informed consent of patients and agreed with the Ethics Committee of Renmin Hospital of Wuhan University. The serum for 8 ALF subjects from the Department of Infectious Diseases, Renmin Hospital of Wuhan University was collected. There were also 8 healthy volunteers who were selected to collect serum as control group. The diagnosis of liver failure complies with the "European Association for the Study of the Liver (EASL) Clinical Practical Guidelines on the management of acute (fulminant) liver failure". ${ }^{16}$ The experimental procedures of the metabolite examination were performed according to the following steps: metabolite extraction, quality control (QC) preparation, sample liquid chromatograph-mass spectrometer/mass spectrometer (LC-MS/MS) analysis, data processing, and quantitative analysis. The human experiment ethics number was WDRY-2021-K016 (approved February 9, 2021).

\section{Regents}

HDAC6 inhibitor Ricolinostat (ACY-1215) was purchased from Selleck (Houston, USA, Cat. No. S8001). D-Galactosamine (D-Gal, Cat. No. G1639) and LPS (Cat. No. L4391) were purchased from Sigma (St. Louis, USA). Dulbecco's modified Eagle's medium (DMEM, Cat. No. 11995065) and fetal bovine serum (FBS, Cat. No. 12483020) were purchased from Gibico (Grand Island, USA). HIF1 $\alpha$ (Cat. No. E-EL-M0687), IL-1 $\beta$ (Cat. No. E-EL-M0037), IL-6 (Cat. No. E-EL-M0044), and TNF- $\alpha$ (Cat. No. E-EL-M0049) enzyme linked immunosorbent assay (ELISA) kits were purchased from Elabscience (Wuhan, China). Rabbit anti-mouse HDAC6 (Cat. No. 7612S) and NLRP3 (Cat. No. 15101S) antibodies were purchased from Cell Signaling Technology (Danvers, USA). Rabbit anti-mouse inducible nitric oxide synthase (iNOS, Cat. No. 18985-1-AP) and CD68 (Cat. 
No. 28058-1-AP) antibodies were purchased from Proteintech (Wuhan, China). Rabbit anti-mouse DDX3X (Cat. No. A5637) and DNMT1 (Cat. No. A19679) antibodies were purchased from ABclonal (Wuhan, China). The fluorescent secondary antibodies with FITC (Cat. No. GB22303) or cy3 (Cat. No. GB21303) tag were obtained from Servicebio (Wuhan, China). Adenosine triphosphate (ATP) content (micro method, Cat. No. BC0305), $\alpha$ Ketoglutarate activity (Cat. No. BC4704), and mitochondrial Isocitrate content (Cat. No. BC2165) detection kits were purchased from Solarbio (Wuhan, China). Citrate content detection kit was purchased from BioAssay Systems (CA, USA, Cat. No. ECIT-100). Oxaloacetate detection kit (Cat. No. KA0867), Succinate Assay Kit (Cat. No. KA3955), and Malate Assay Kit (Cat. No. KA4571) were purchased from Abnova (Taipei, China). Mouse isocitrate dehydrogenase [NADP] cytoplasmic (IDH1) ELISA kit was purchased from Abbexa (Cambridge, UK, Cat. No. abx515558). Malate dehydrogenase 1(MDH1) ELISA kit was purchased from ELK Biotechnology (Wuhan, China, Cat. No. ELK7808). The

Annexin V-phycoerythrin/7-aminoactinomycin D (Annexin V-PE/7AAD) apoptosis kit was obtained from BD Biosciences (CA, USA, Cat. No. 559763).

\section{Mice Group, Drug Administration and Quantitative Proteomic Analysis}

The animal experiment was approved by the Institutional Animal Care and Use Committee of Renmin Hospital of Wuhan University. It complied with the requirements of the National Institutes of Health guidelines. The animal experiment ethics number was WDRM (Welfare) 20181018 (approved on October 26, 2018). Twenty-four male C57BL/6 mice (6-week-old, weighing 18-22 g) were provided by Hubei Animal Experimental Center (Wuhan, China). They were housed in the animal experiment center in Renmin Hospital of Wuhan University with relative humidity of $50 \% \pm 15 \%$, temperature of $25 \pm 2{ }^{\circ} \mathrm{C}$ and $12 \mathrm{~h}$ light/dark cycle. The mice were randomly divided into control group, LPS/D-Gal (model) group and ACY1215 group. There were 8 mice in each group. After one week of adaptive feeding, the ACY-1215 group mice were intraperitoneally injected with ACY-1215 (25 mg/kg) before building the ALF model. The LPS/D-Gal group and control group mice were given the same amount of saline. Then the ALF model mice were intraperitoneally injected with LPS $(100 \mu \mathrm{g} / \mathrm{kg})$ and D-gal $(400 \mathrm{mg} / \mathrm{kg})$ for
$24 \mathrm{~h}$. The ACY-1215 group mice were in the same way intraperitoneally injected with LPS/D-gal for $24 \mathrm{~h}$. After anesthesia and femoral artery blood collection, mice were sacrificed by spinal dislocation. The livers were separated accompanied with serum collection and stored at $-80{ }^{\circ} \mathrm{C}$ for testing. According to our previous study, the liver protein expression profiles in control group, LPS/D-Gal group and ACY-1215 group were detected by tandem mass tags/isobaric tag for relative absolute quantitation (TMTT/ iTRAQ) technology. ${ }^{17}$

\section{Cell Culture and Intervention}

Mouse macrophage RAW264.7 was purchased from China Center for Type Culture Collection (Wuhan University). The cells were cultured in a cell culture incubator at $37^{\circ} \mathrm{C}$ with $5 \% \mathrm{CO}_{2}$ concentration in DMEM containing $10 \%$ FBS. The cells were divided into control group, LPS (model) group and ACY-1215 group. Cells in ACY-1215 group were treated with ACY-1215 (10 $\mu \mathrm{mol} / \mathrm{L})$ for $2 \mathrm{~h}$. Then the LPS group and ACY-1215 group cells were stimulated with LPS ( $1 \mu \mathrm{g} / \mathrm{mL})$ for $24 \mathrm{~h}$. The supernatants and cells were harvested for subsequent detections.

\section{Measurement of Energy Metabolites and Cytokines in Liver Tissue and Cell Lysates}

The liver tissue and cells were homogenized and centrifuged. Then the supernatant was collected. The content of IDH1, MDH1, HIF1 $\alpha$, TNF- $\alpha$, IL-1 $\beta$ and IL-6 in the cell and liver tissue was measured by ELISA kit. The operation was strictly performed according to the instructions of the kits. The ATP, Oxaloacetate, $\alpha$ Ketoglutarate, Citrate, L-Malate, Isocitrate, and Succinate contents were tested strictly according to the instructions of the assay kits.

\section{Pathological Changes of Liver Tissue and Liver Function Test}

Mice liver tissues were fixed in $4 \%$ paraformaldehyde solution, embedded in conventional paraffin, sliced, and stained with hematoxylin-eosin (HE) staining. The structure of liver tissue was observed under a light microscope. The levels of alanine aminotransferase (ALT), aspartate aminotransferase (AST) and total bilirubin (TBIL) in serum were measured by the fully automated aeroset chemistry analyzer from Abbott Co. Ltd (MA, USA). 
Detection of Protein in Tissues and Cells by Immunofluorescence (IF)

Liver tissue and slides with RAW264.7 cells were fixed in 4\% paraformaldehyde. After dehydration of liver tissue, paraffin was embedded and sectioned. Liver tissue sections were dewaxed with xylene, rehydrated with gradient ethanol, and washed with PBS. Liver tissue sections and cell slices were then placed in 5\% bovine serum albumin and incubated. $0.3 \%$ Triton $\mathrm{X}$ was punched. The slices were incubated with rabbit anti-mouse primary antibodies HDAC6 (1: 100), NLRP3 (1: 100), iNOS (1: 100), CD68 (1: 100), DDX3X (1: 100), and DNMT1 (1: 100) overnight. After being washed with PBS, the slices were incubated with fluorescent secondary antibody with FITC or cy3 tag (1:200) incubated at room temperature in the dark. Then, they were stained with DAPI at room temperature and observed under the fluorescence microscope. The fluorescence intensity was analyzed with ImageJ software. For the statistics of immunofluorescence in the in vivo experiment, three tissue specimens were randomly selected, three sections were selected from each specimen, and five discontinuous fields of view were selected in each section. The fluorescence intensity of the stained cells was then evaluated, and their averages were subsequently calculated. For the statistics of immunofluorescence in the in vitro experiment, we selected three different repeated experiments, each time making three slices, selecting five discontinuous different fields of view for each slice under the microscope. The relevant description has been added in the article.

\section{Cell Apoptosis Detection}

The cell apoptosis rates were detected by flow cytometry with Annexin V-PE/7AAD apoptosis kit. After the $10^{5}$ cells were processed to a 6-well plate, they were collected for staining. The cells were added with $400 \mu \mathrm{L}$ buffer solution, $5 \mu \mathrm{L}$ Annexin V-PE, and $5 \mu \mathrm{L} 7 \mathrm{AAD}$, then incubated for $15 \mathrm{~min}$ at $37^{\circ} \mathrm{C}$ away from light. Flow cytometry (BD, USA) was used to count the cell apoptosis rate.

\section{Statistical Analysis}

The measurement data were expressed as mean \pm standard deviation and analyzed with SPSS16.0 statistical software. After the homogeneity test for variance, the differences between two groups were analyzed using a Student's $t$-test, and the differences between groups were evaluated using one-way analysis of variance (ANOVA). $P<0.05$ was considered statistically significant. The Pearson correlation analysis method was used to analyze the correlation coefficient between different metabolites. The Pearson correlation coefficient value $\mathrm{R}$ was between -1 and +1 . The correlation coefficient $\mathrm{R}$ between metabolites was represented by color and circle, where $\mathrm{R}>0$ represented a positive correlation, and it was represented by red. $\mathrm{R}<0$ indicates a negative correlation, which was represented by blue. The larger and darker the circles, the more relevant they were.

\section{Results}

\section{Characteristics of Energy Metabolism for ALF Patients}

The serum samples from 8 normal volunteers and ALF patients were separated and targeted metabolomics-quantitative analysis of energy metabolites was applied to explore the characteristics for energy metabolism. As shown in Figure 1A, there was 30 energy metabolism related products examined.

Among these, the expression levels of Isocitrate, L-Lactate, L-Malate, Succinate, 3-phosphoglycerate, ADP, $\alpha$-Ketoglutarate, ATP, Citrate, NAD, NADPH, Oxaloacetate, Phosphoenolpyruvate, UDPglucose were significantly different between ALF patients and normal people (Figure 1B). Isocitrate, L-Lactate, L-Malate, and Succinatewere expressed more highly in ALF patients compared with normal volunteers $(P<0.05)$. 3-phosphoglycerate, ADP, $\alpha$-Ketoglutarate, ATP, Citrate, NAD, NADPH, Oxaloacetate, Phosphoenolpyruvate, and UDPglucose had lower expression in ALF patients compared with normal volunteers $(P<0.05)$. More importantly, the ATP level in ALF patients was only 0.09 times that of normal volunteers $(P=0.012)$. As shown in Figure 1C, the expression for Oxaloacetate, $\alpha$ Ketoglutarate, and Citrate was positively correlated with ATP. On the contrary, the expression for L-Malate, Isocitrate, and Succinate was negatively correlated with ATP. Therefore, the basis of this experimental part suggested that the occurrence of ALF was accompanied by a significant energy metabolism disorder. This is consistent with the typical energy metabolism characteristics of M1 macrophages. However, as shown in Figure 1D, the metabolic characteristics of M1 macrophages in the liver during ALF deserved further study, to see whether it was possible to reverse the differentiation of M1 macrophages by 

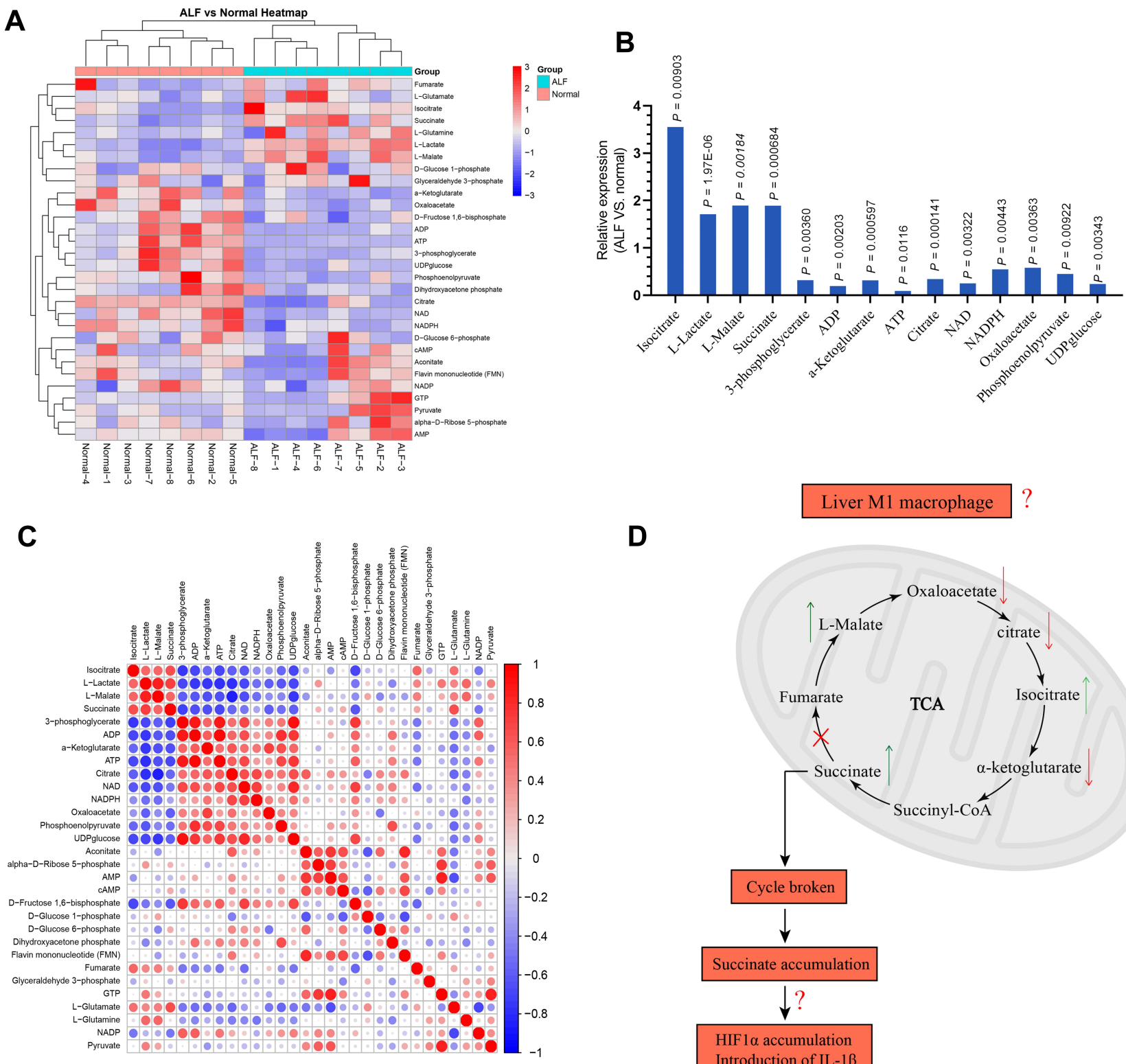

D

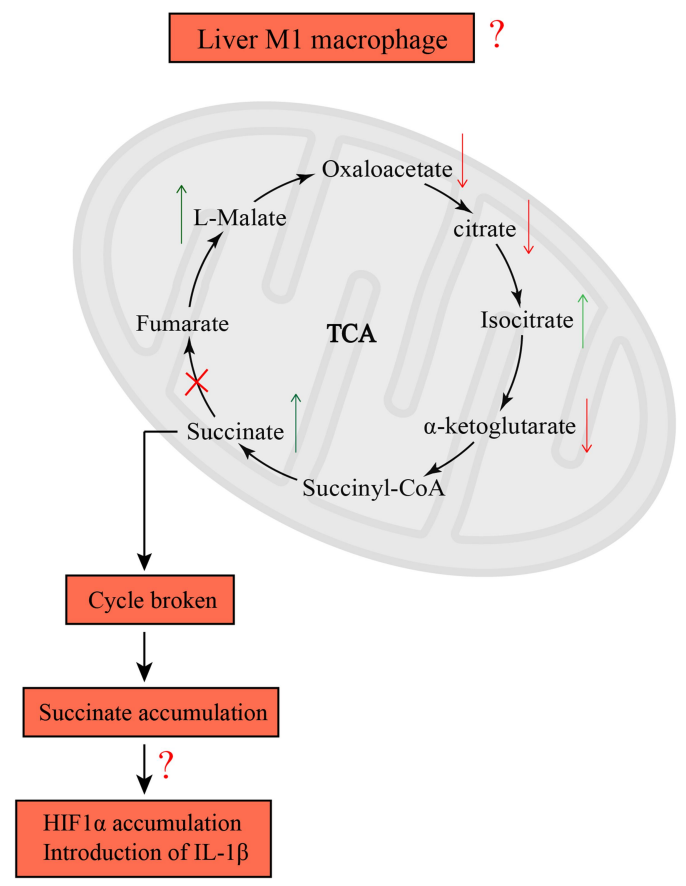

Figure I (A) Characteristics of energy metabolism for ALF patients. (B) The expression levels of L-Lactate, L-Malate, Isocitrate, Succinate, 3-phosphoglycerate, ADP, $\alpha$ Ketoglutarate, ATP, Citrate, Oxaloacetate, NAD, NADPH, Phosphoenolpyruvate, and UDPglucose were significantly different between ALF patients and normal volunteers. (C) Based on the Pearson correlation analysis method, the correlation coefficient between different metabolites was calculated. The correlation between the different metabolites was displayed in the form of a correlation coefficient matrix heat map, and the expression of each metabolite was replaced by the ratio of the relative expression of the ALF group to the relative expression of the normal group. This matrix chart shows the correlation between significantly different metabolites. The Pearson correlation coefficient value $\mathrm{R}$ was between $-\mathrm{I}$ and $+\mathrm{I}$. The correlation coefficient $\mathrm{R}$ between metabolites was represented by color and circle, where $\mathrm{R}>0$ represents a positive correlation, and it was represented by red. $R<0$ indicates a negative correlation, which was represented by blue. The larger and darker the circles, the more relevant they were. It was found that the expression for $\alpha$-Ketoglutarate, Citrate, and Oxaloacetate was positively correlated with ATP. The expression for L-Malate, Isocitrate, and Succinate was negatively correlated with ATP. (D) Hypothesis diagram of MI macrophage energy metabolism during ALF process.

affecting the energy metabolism, thereby reducing the inflammatory response to alleviate ALF.

\section{The Effect of ACY-I2I5 on LPS/D-Gal Induced ALF Mice}

LPS combined with D-Gal was firstly used to intervene in mice to build an ALF model. Then the mice model was treated with HDAC6 inhibitor ACY-1215. As shown in Figure 2A, HE staining results revealed that the hepatocytes in the control group were neatly arranged. In the LPS/D-Gal group, hepatic lobular structures were disordered accompanied with inflammatory cell infiltration and hepatocytes necrosis. The pathological changes in the ACY-1215 group were reduced 
A

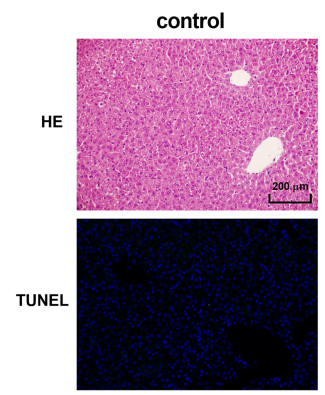

LPS/D-Gal

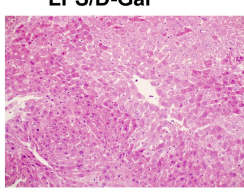

(1)
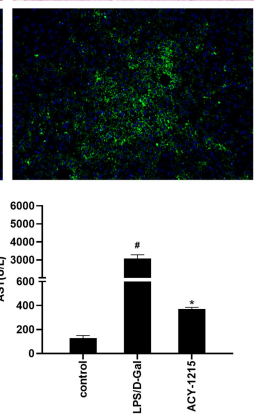

C

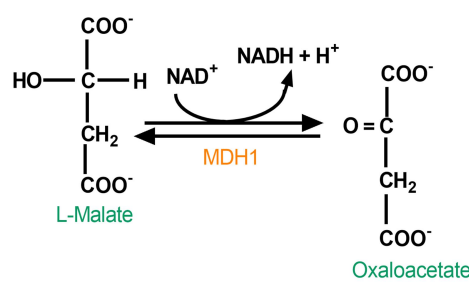

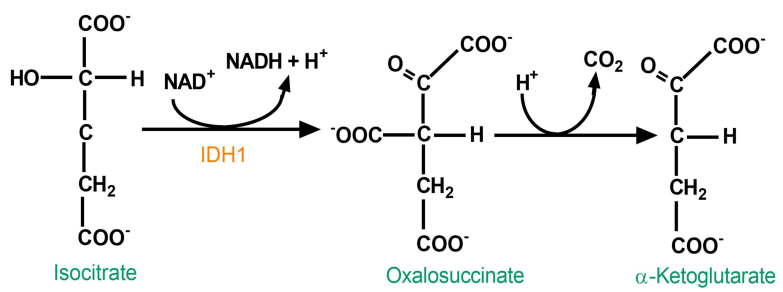
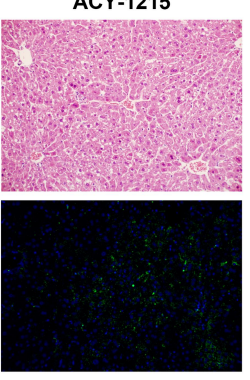

B

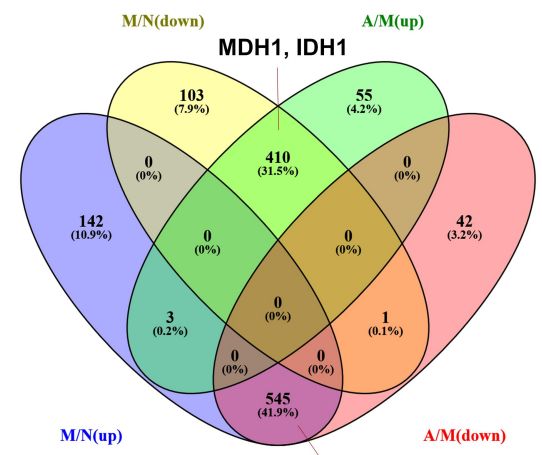

DNMT1
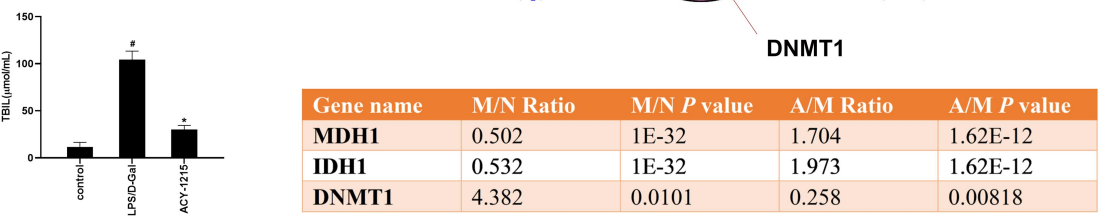

D
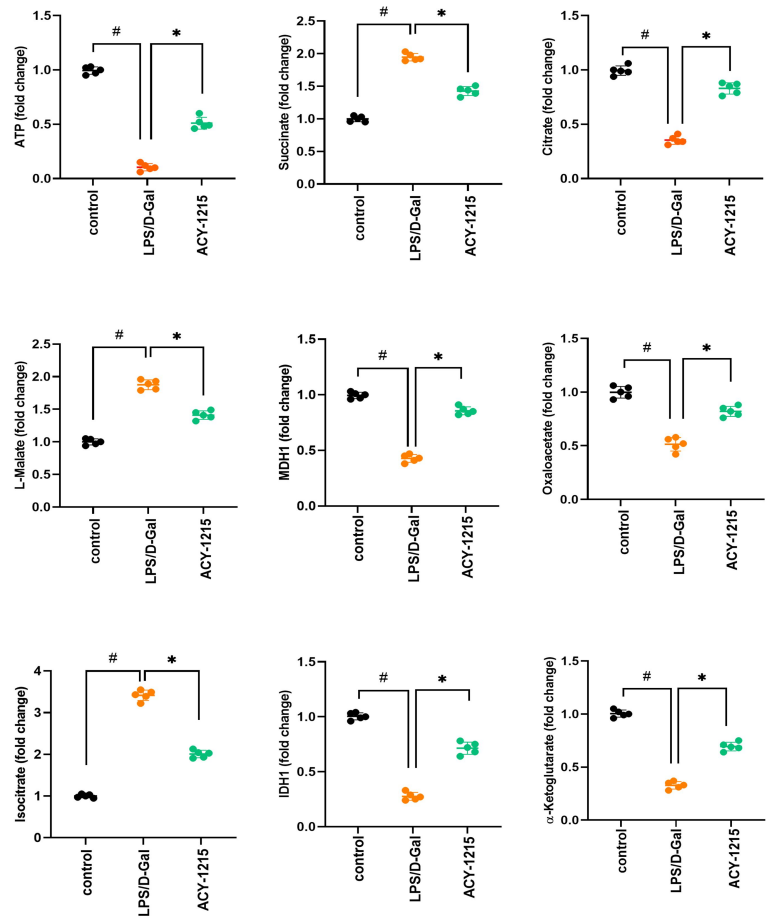

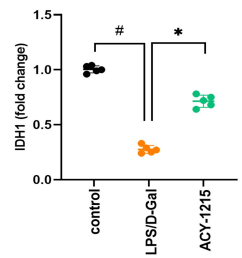

Figure 2 The effect of ACY-12I5 on LPS/D-Gal induced ALF mice. (A) HE staining was used to detect histopathological changes in liver. TUNEL staining was used to detect the cell apoptosis level. The serum levels of ALT, AST, and TBIL were detected. (B) Venn diagram showing the protein quantitative sequencing. (A) ACY-I2I5 intervention group. M: Model group. N: Control group. M/N (up/down) represented the protein whose expression level was increased (decreased) in the model group compared with the normal group. A/M (up/down) represented the protein whose expression level was increased (decreased) in the ACY-1215 intervention group compared with the model group. The table showed different expression levels of MDHI, IDHI and DNMTI in different mice groups. (C) The substrate and product of MDHI were L-Malate and

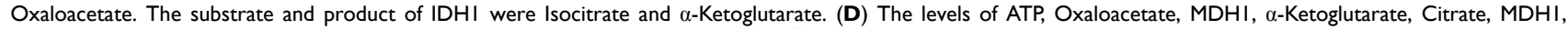
L-Malate, Isocitrate and Succinate in liver were tested. Data are shown as mean \pm SD. ${ }^{\#} P<0.05$, compared with the control group. ${ }^{*} P<0.05$, compared with the LPS/D-Gal group.

compared with the LPS/D-Gal group. TUNEL staining results showed that the apoptosis level in LPS/D-Gal group liver was significantly increased compared with the control group. However, the apoptosis level in ACY1215 group liver was significantly reduced compared with the LPS/D-Gal group. For the liver function, compared with the control group, the serum levels of ALT, AST, and TBIL in the LPS/D-Gal group were significantly increased $(P<0.05)$. Compared with the LPS/D-Gal group, the serum levels of ALT, AST, and TBIL in ACY-1215 group were significantly reduced $(P<0.05)$. 


\section{The Mice Liver Protein Expression Profiles Among Mice Groups Related with TCA}

In order to exactly find out which regulatory enzymes in the TCA process were involved in the activation of M1 macrophages during ALF, and whether the expression of these enzymes can be regulated after ACY-1215 intervention, we used LC-MS/MS analysis technology to establish the expression profiles of all mice liver protein molecules for control, LPS/D-Gal, and ACY-1215 mice groups. As the Venn diagram shows in Figure 2B, the protein quantitative sequencing results showed that only MDH1 and IDH1 in TCA were differently expressed among normal, LPS/D-Gal and ACY-1215 groups. The expression of MDH1, IDH1, DNMT1 in the LPS/D-Gal group was separately 0.502 -fold change $(P=1.0 \mathrm{E}-32), 0.532$-fold change $(P=1.0 \mathrm{E}-32)$, and 4.382 -fold change $(P=$ $0.0101)$ versus the control group. However, the expression of MDH1, IDH1, DNMT1 in ACY-1215 group was separately 1.704-fold change $(\mathrm{P}=1.62 \mathrm{E}-12), 1.973$-fold change $(P=1.62 \mathrm{E}-12)$, and 0.258 -fold change $(P=$ 0.00818 ) versus the LPS/D-Gal group.

\section{The Effect of ACY-1215 on Energy Enzymes and Metabolites for ALF Mice}

As shown in Figure $2 \mathrm{C}$, the substrate and product of MDH1 were L-Malate and Oxaloacetate. The substrate and product of IDH1 were Isocitrate and $\alpha$-Ketoglutarate. Meanwhile, Succinate and Citrate during the TCA process had been reported to have pro-inflammatory and antiinflammatory effects, respectively. ${ }^{18}$ In order to verify the effect of ACY-1215 on liver energy metabolism in ALF mice, ATP, Succinate, Citrate, L-Malate, MDH1, Oxaloacetate, IDH1, and $\alpha$-Ketoglutarate were detected in liver tissue homogenates. As shown in Figure 2D, the levels of ATP, Citrate, MDH1, Oxaloacetate, MDH1, $\alpha-$ Ketoglutarate in the LPS/D-Gal group were decreased compared with the control group $(P<0.05)$. The levels of Succinate, L-Malate, and Isocitrate increased in the LPS/D-Gal group compared with the control group $(P<$ 0.05). After intervention with ACY-1215, the levels of ATP, Citrate, MDH1, Oxaloacetate, MDH1, and $\alpha$ Ketoglutarate increased compared with the LPS/D-Gal group $(P<0.05)$. The levels of Succinate, L-Malate, and Isocitrate decreased compared with the LPS/D-Gal group $(P<0.05)$.

\section{The Effect of ACY-I2I5 on DNMTI, DDX3X/NLRP3 Pathway and Cytokines in ALF Mice}

As shown in Figure 3A and B, the levels of HDAC6, DNMT1 and NLRP3 increased in the LPS/D-Gal group compared with the control group $(P<0.05)$. The level of DDX3X was decreased in LPS/D-Gal group compared with the control group $(P<0.05)$. After intervention with ACY-1215, the levels of HDAC6, DNMT1 and NLRP3 decreased compared with the LPS/D-Gal group $(P<0.05)$. The level of DDX3X increased when compared the LPS/D-Gal group $(P<0.05)$.

As shown in Figure 4A-D, when compared with control group, the cytokine levels of HIF $1 \alpha$, TNF- $\alpha$, IL-6 and IL-1 $\beta$ increased in the LPS/D-Gal group $(P<0.05)$. However, the levels of HIF $1 \alpha$, TNF- $\alpha$, IL- 6 and IL-1 $\beta$ decreased in the ACY-1215 group compared with the LPS/D-Gal group $(P<0.05)$. In order to further explore the characteristics of energy metabolism of M1 macrophages in the liver, we first detected the distribution characteristics of M1 macrophages in mice liver for the LPS/ D-Gal group and ACY-1215 group. Referring to our previous research and the reported study, the biomarkers CD68 and iNOS are widely used to study the effects of M1 macrophages in liver. ${ }^{19,20}$ As shown in Figure 4E, the marker proteins of M1 macrophages iNOS and CD68 in the LPS/D-Gal group increased compared with the control group $(P<0.05)$. When compared with the LPS/D-Gal group, the level of iNOS and CD68 had decreased in the ACY-1215 group $(P<0.05)$.

\section{The Effect of ACY-I2I5 on Energy Metabolite in LPS Induced RAW264.7 Cells}

However, for M1 macrophages and ACY-1215 intervened M1 macrophages, whether the energy metabolism process also meets the above characteristics required further verification in in vitro experiments. Therefore, this experimental part used LPS to induce RAW264.7 cells to differentiate into M1 macrophages, and further used ACY-1215 for intervention. As shown in Figure 5A, the levels of ATP, Citrate, MDH1, Oxaloacetate, MDH1, and $\alpha$-Ketoglutarate in the LPS/D-Gal group decreased compared with the control group $(P<0.05)$. The levels of Succinate, L-Malate, and Isocitrate increased in the LPS group compared with the control group $(P<0.05)$. After intervention with ACY- 


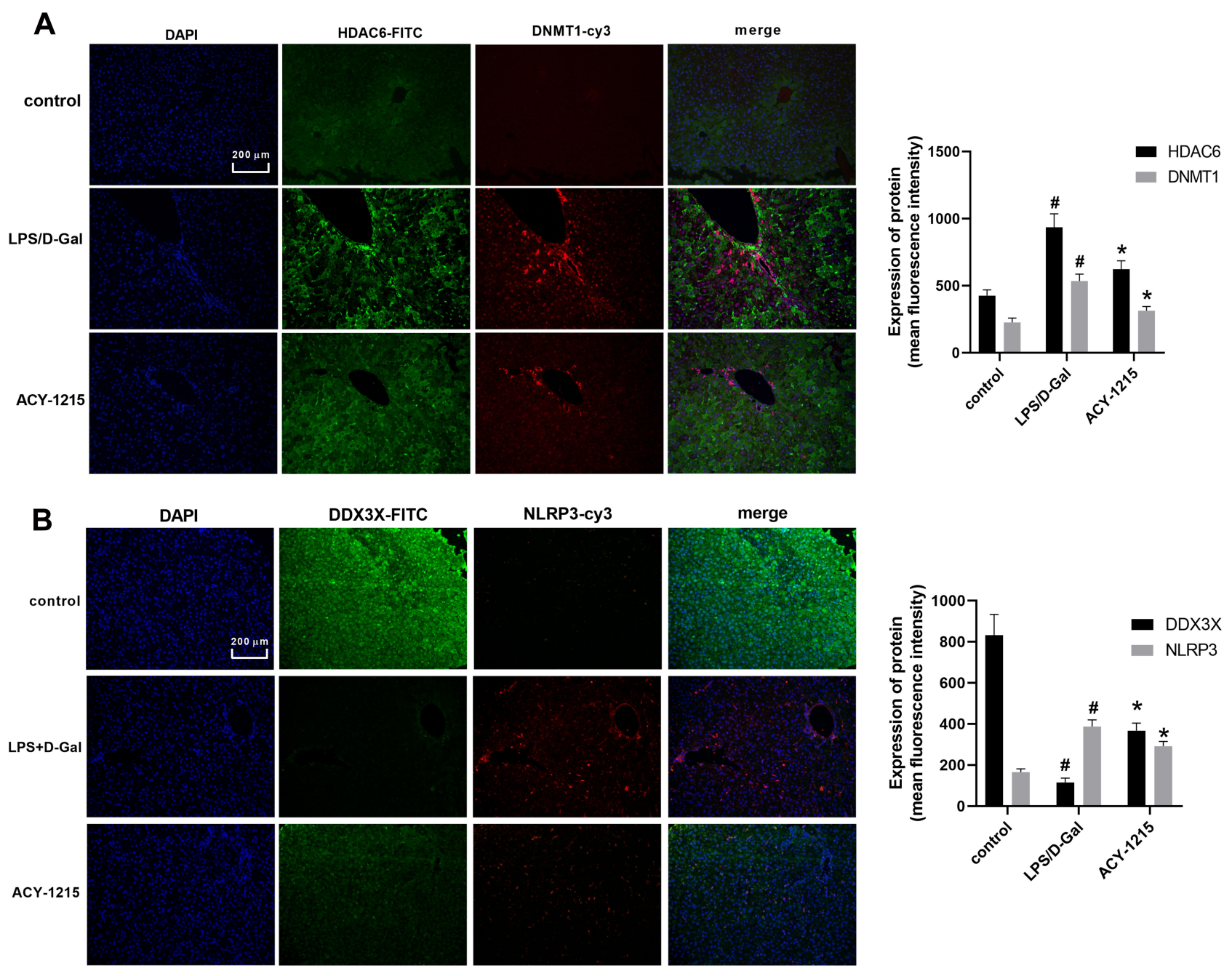

Figure 3 The effect of ACY-I2I5 on Acetyl-DNMTI, DDX3X/NLRP3 pathway and cytokines in ALF mice. (A) The level of HDAC6 and DNMTI was detected by immunofluorescence. (B) The level of NLRP3 and DDX $3 X$ was detected by immunofluorescence. Data are shown as mean \pm SD. ${ }^{\# P}<0.05$, compared with control group. $* P<0.05$, compared with LPS/D-Gal group.

1215, the levels of ATP, Citrate, MDH1, Oxaloacetate, $\mathrm{MDH} 1$, and $\alpha$-Ketoglutarate increased compared with the LPS group $(P<0.05)$. The levels of Succinate, L-Malate, and Isocitrate decreased compared with the LPS group $(P<0.05)$.

\section{The Effect of ACY-1215 on Acetyl- DNMTI, DDX3X/NLRP3 Pathway and Cytokines in LPS Induced RAW264.7 Cells}

As shown in Figure 5B-E, the levels of DNMT1 and NLRP3 was increased $(P<0.05)$. The level of DDX3X decreased in the LPS group compared with the control group $(P<0.05)$. After intervention with ACY-1215, the levels of DNMT1 and NLRP3 decreased $(P<0.05)$. The level of DDX3X increased compared with the LPS group $(P<0.05)$. As shown in Figure 5F, when compared with the control group, the cell apoptosis rate was increased in the LPS group $(P<0.05)$. However, the cell apoptosis rate was decreased in the ACY-1215 group compared with the LPS group $(P<0.05)$. As shown in Figure 5G, compared with the control group, the cytokine levels of HIF $1 \alpha$, TNF$\alpha$, IL-6 and IL-1 $\beta$ increased in the LPS group $(P<0.05)$. However, the levels of HIF $1 \alpha$, TNF- $\alpha$, IL-6 and IL-1 $\beta$ decreased in the ACY-1215 group compared with the LPS group $(P<0.05)$.

\section{Discussion}

Regarding the pathogenesis of acute liver failure, most scholars currently support the "two-hit" theory. ${ }^{21}$ Studies have shown that ALF patients have severe 
A

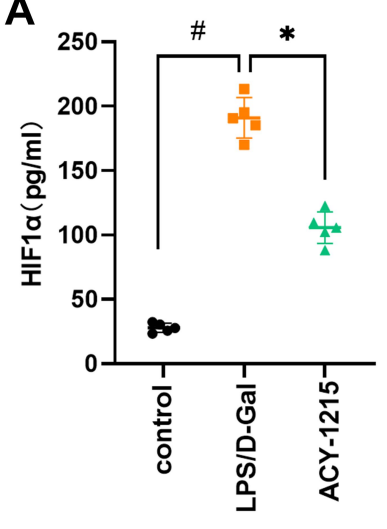

B

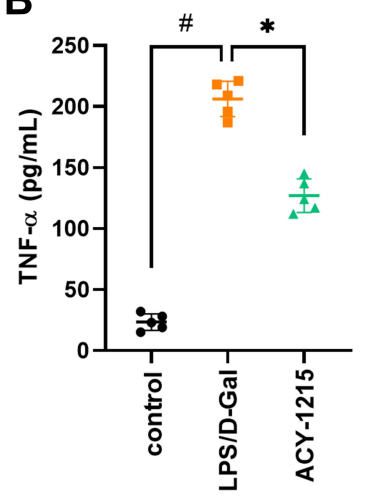

E

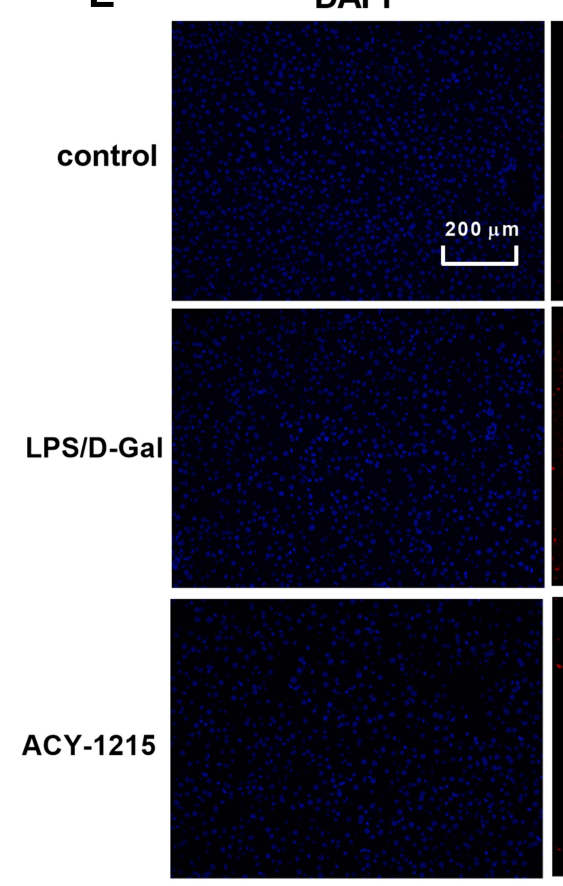

C

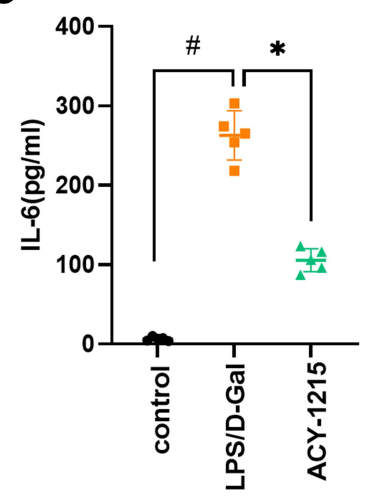

CD68-FITC
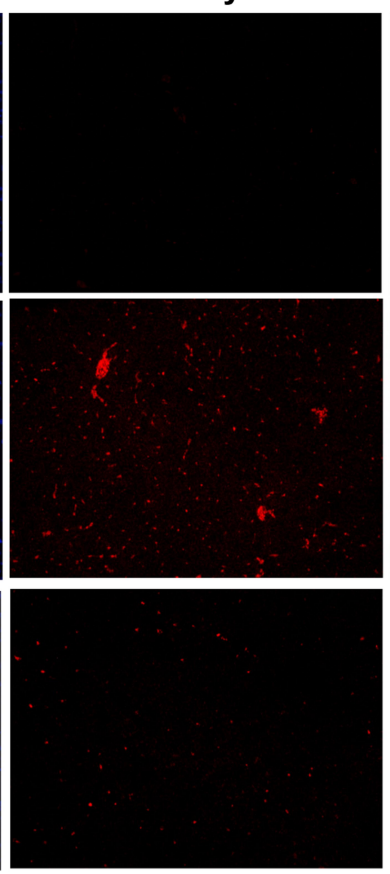

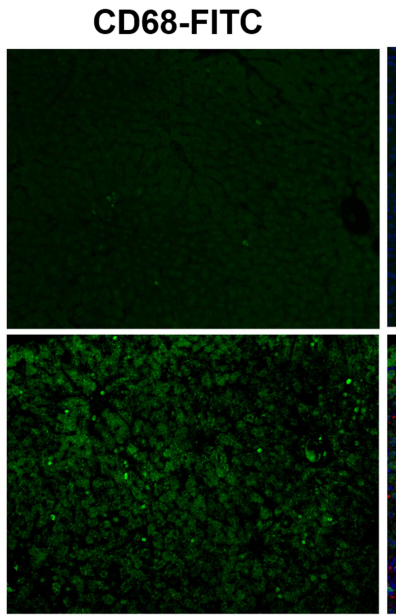

D

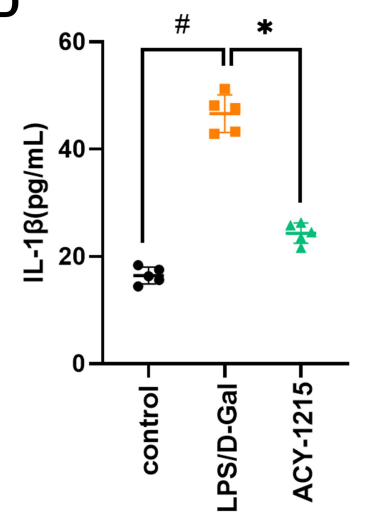

merge
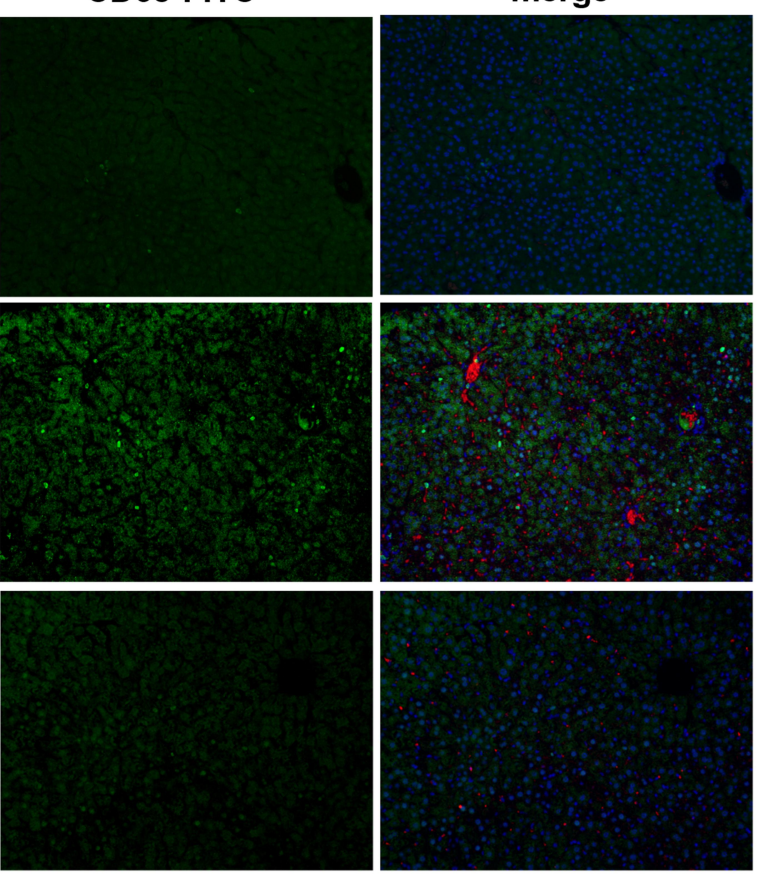

Figure 4 The effect of ACY-I2I5 on cytokines and marker proteins of MI macrophages in ALF mice. (A-D) The levels of HIFI $\alpha$, TNF- $\alpha$, IL-6, and IL-I $\beta$ were tested by ELISA kits. (E) The levels of iNOS and CD68 were detected by immunofluorescence. Data are shown as mean \pm SD. ${ }^{*} P<0.05$, compared with the control group. ${ }^{*} P<0.05$, compared with the LPS/D-Gal group.

intestinal micro-ecological imbalances. The intestinal wall barrier function has been impaired, inducing intestinal bacteria and endotoxin translocation. ${ }^{22}$ Bacterial translocation can cause endotoxemia and various infections, such as spontaneous peritonitis. LPS can induce localized non-specific hypersensitivity reactions, causes liver microcirculation disorders and hepatocytes damage. ${ }^{5,23}$ LPS can also stimulate liver Kupffer cells (KC) to differentiate into M1 macrophages. The M1 macrophages release cytokines (TNF$\alpha$, IL-1, IL-6) and cause further necrosis of hepatocytes. $^{24}$
It has been discovered that macrophage activation is accompanied by changes in energy metabolism levels. ${ }^{8}$ The activation of M1 macrophages requires energy through glycolysis. Under the catalysis of pyruvate dehydrogenase kinase 1 (PDK1), glucose is broken down, which promotes the conversion of pyruvate to lactate and inhibits TCA, and activates HIF 1a. ${ }^{8}$ At the same time, oxidative respiration of cells weakens. The production of reactive oxygen species (ROS) and nitric oxide (NO) is increased. During the process of M1 macrophages obtaining energy, TCA cycles are broken, resulting in a large amount of metabolite (like Succinate) 
A

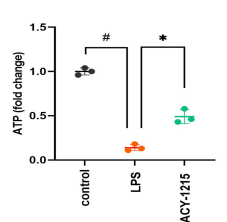

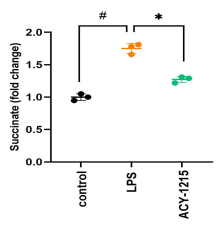

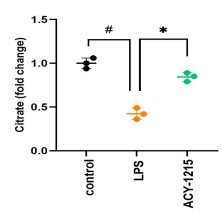

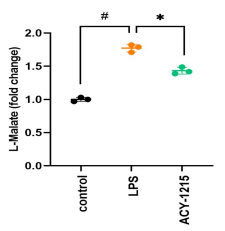

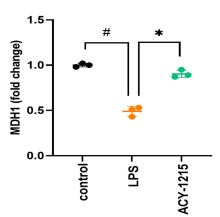

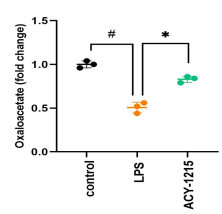

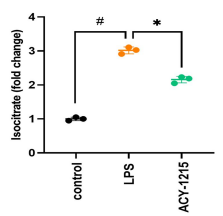

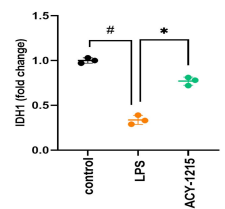

C

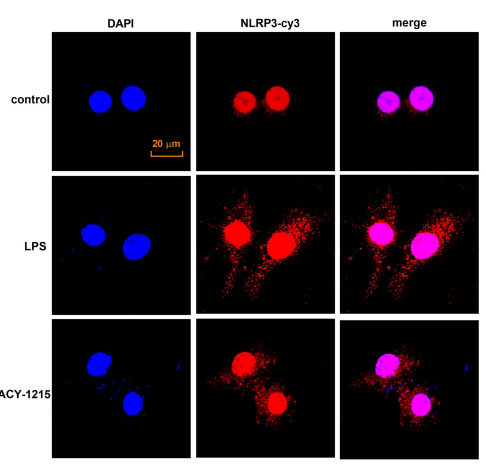

F
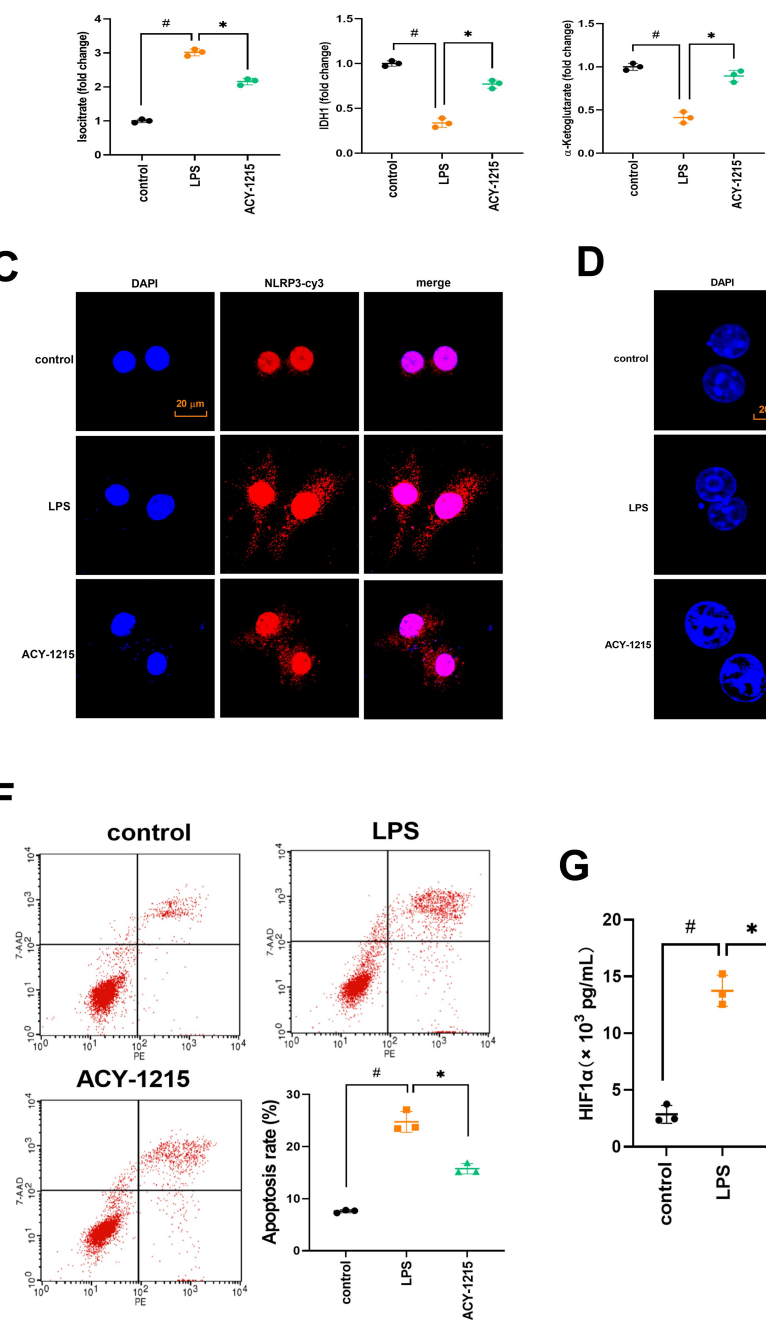

D

G

B
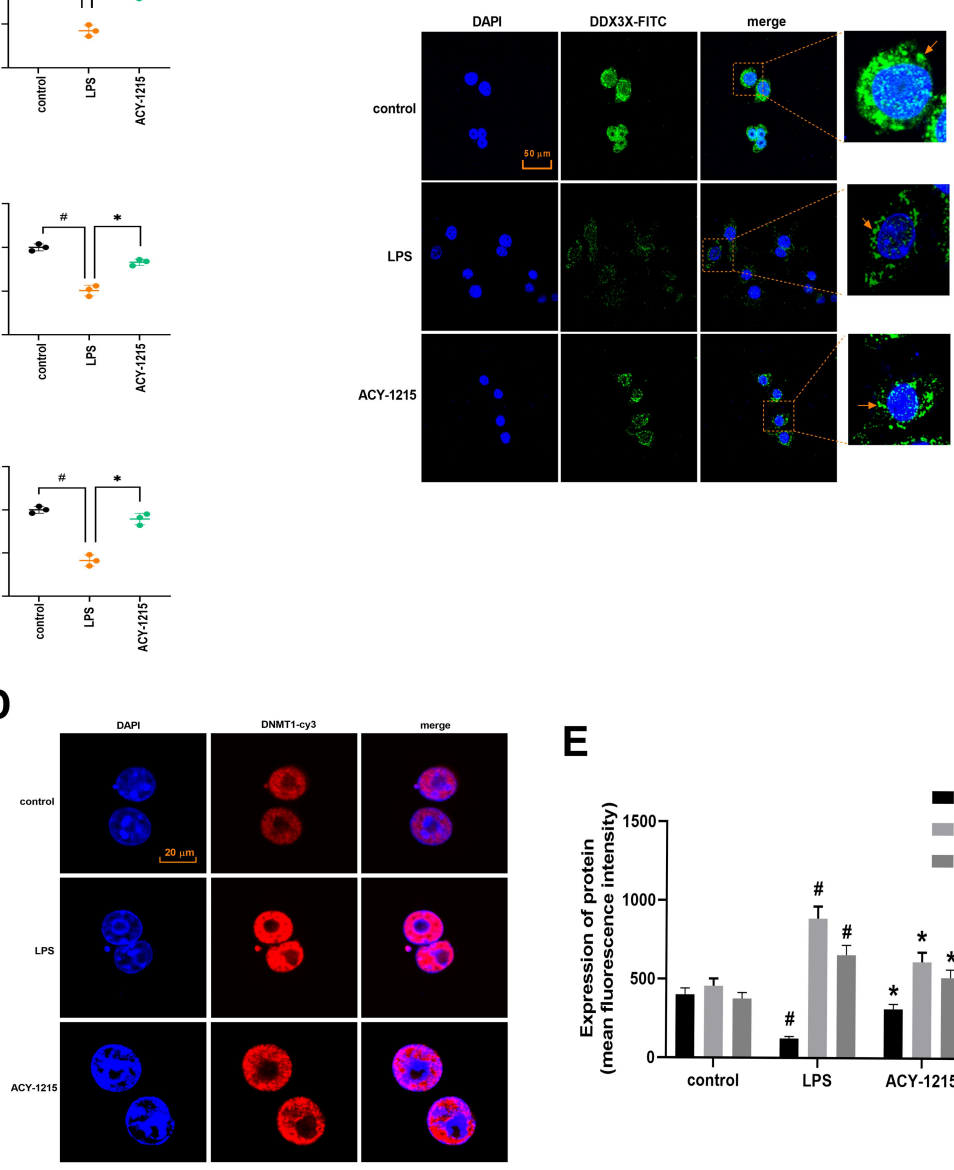

E
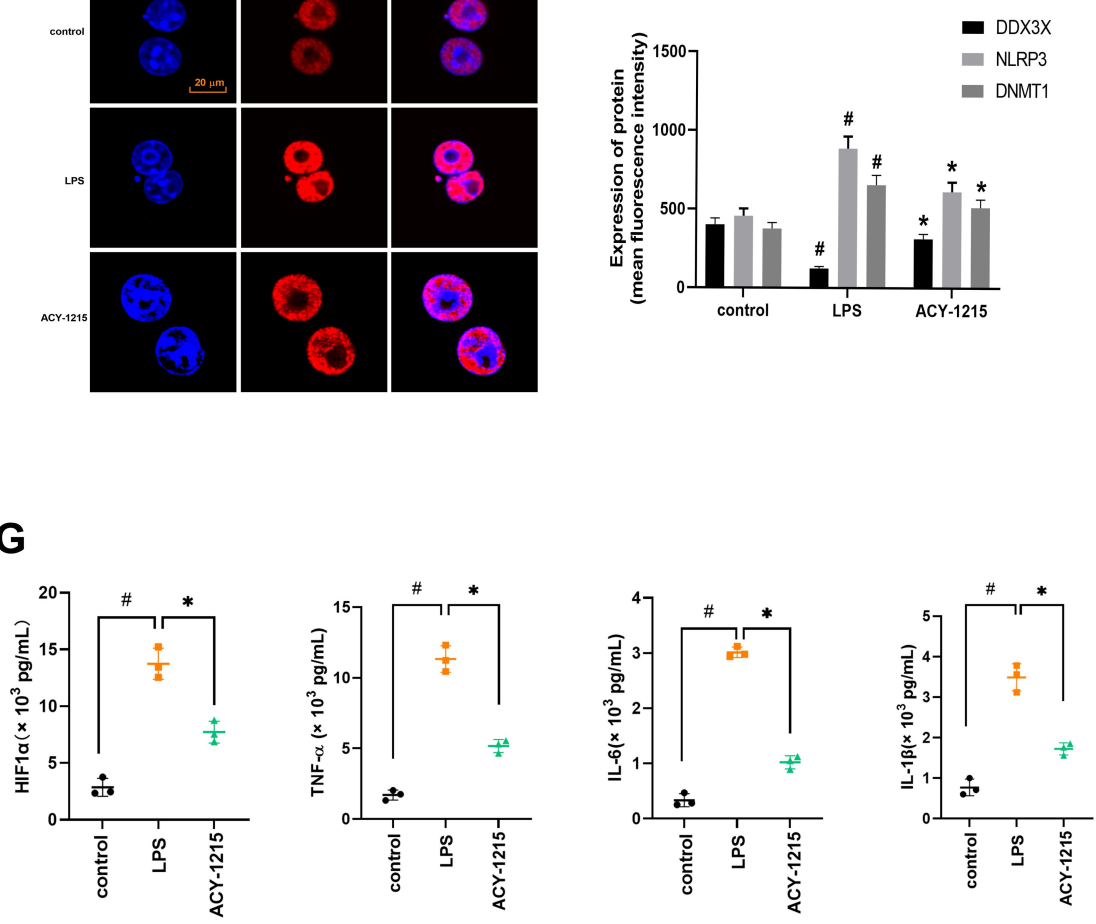

Figure 5 The effect of ACY-1215 on energy metabolite in LPS induced RAW264.7 cells. (A) The levels of ATP, Oxaloacetate, MDHI, $\alpha-$ Ketoglutarate, Citrate, MDHI, L-Malate, Isocitrate, and Succinate in RAW264.7 cells was tested. (B-D) The levels of DDX3X, NLRP3, and DNMTI were detected by immunofluorescence. (E) A histogram was used to show the protein level of DDX3X, NLRP3 and DNMTI. (F) Cell apoptosis was detected by flow cytometry. (G) The levels of HIFI $\alpha$, TNF- $\alpha$, IL-6, and IL-I $\beta$ in cell were tested by ELISA kits. Data are shown as mean \pm SD. ${ }^{\#} P<0.05$, compared with the control group. $* P<0.05$, compared with the LPS group.

accumulation. ${ }^{25}$ It is reported that Succinate has a proinflammatory effect and Citrate has an anti-inflammatory effect during macrophage metabolic reprogramming. ${ }^{26,27}$ Therefore, the mitochondrial glycolysis pathway of M1 macrophages is a key target for the acute inflammatory response of ALF.

Epigenetics, characterized by changes in the expression of genes that occur through post-translational modification of 
protein without altering the underlying genetic DNA sequence, has a complex regulatory mechanism for liver inflammation. $^{28}$ These post-translational modifications include histone acetylation, DNA methylation, sumoylation, and ubiquitination. HDACs could regulate protein expression through modifying the combined histone molecules. ${ }^{29}$ In our previous studies, the HDAC6 inhibitor ACY-1215 was verified to alleviate ALF through reducing liver inflammatory response. $^{12-14}$ As an NLRP3 upstream molecule, DDX3X induces inflammasome activation when it binds to NLRP3 under cellular stress. ${ }^{15}$ Activation of inflammatory bodies can cause disorders of lipid metabolism in macrophages. ${ }^{30}$ However, whether the DDX3X/NLRP3 signaling pathway can participate in the energy metabolism during the activation of M1 macrophages in ALF, and whether ACY-1215 can promote the energy metabolism of M1 macrophages by regulating the DDX3X/NLRP3 pathway, are rarely reported and worth further studies.

As shown in our experiment results, the serum samples from 8 normal volunteers and ALF patients were first separated to explore the characteristics for energy metabolism. The ATP level in ALF patients was only 0.09 times that of normal volunteers. Meanwhile, Oxaloacetate, $\alpha$ Ketoglutarate, Citrate, L-Malate, Isocitrate, and Succinate were abnormally expressed for ALF patients. The occurrence of ALF was accompanied by a significant energy metabolism disorder. The ALF mice model was then built and treated with HDAC6 inhibitor ACY-1215. The pathological changes, the liver apoptosis level and liver function in the ACY-1215 group were improved compared with the model group. The LC-MS/MS analysis technology was then used to establish the expression profiles of all mice liver protein molecules. It was found that only MDH1 and IDH1 in TCA were differently expressed among the normal, model, and ACY-1215 groups. More importantly, ATP, Succinate, Citrate, L-Malate, MDH1, Oxaloacetate, IDH1, and $\alpha$-Ketoglutarate were also found to have unusual expression and improved in the ACY-1215 group.

The increased marker proteins for M1 macrophages (iNOS and CD68) and cytokines (HIF1 $\alpha$, TNF- $\alpha$, IL-6, and IL-1 $\beta$ ) in the LPS/D-Gal group testified that the macrophages in liver were mainly M1 type during ALF. When treated with ACY-1215, the levels of iNOS and CD68 decreased. Therefore, ACY-1215 may not only improve energy metabolism in vivo, but also inhibit the activation of M1 macrophages. However, for M1 macrophages and ACY-1215 intervened M1 macrophages, whether the energy metabolism process also smet the above characteristics required further verification in in vitro experiments. Therefore, LPS was used to induce RAW264.7 cells to differentiate into M1 macrophages, and further used ACY-1215 for intervention. ATP, Succinate, Citrate, L-Malate, MDH1, Oxaloacetate, IDH1, and $\alpha$-Ketoglutarate were also found to have unusual expression and improved in the ACY-1215 group.

In both the in vitro and in vivo experiments, the levels of DNMT1 and NLRP3 increased in the ALF model group. After intervention with ACY-1215, the levels of DNMT1 and NLRP3 were decreased. However, the level of DDX3X was decreased in the LPS group, which was consistent with the reported study that DDX3X played a protective role in liver Injury. ${ }^{31}$ ACY-1215 could improve the expression of DDX3X and DNMT1. Therefore, ACY1215 regulated the DDX3X/NLRP3 pathway and improved the activation of M1 macrophages. Its core role was that ACY-1215 promoted the expression of DDX3X, IDH1 and MDH1. We speculated that the specific mechanism was to promote the acetylation of lysine sites on molecules for DDX3X, IDH1, and MDH1 by inhibiting the expression of HDAC6, to promote its expression.

It is worth noting that ACY-1215 can also inhibit the expression of DNMT1. Epigenetic regulation of histone acetylation and DNA methylation has not been reported during the ALF process. However, DNA methyltransferase DNMT1 was reported to be closely associated with HDAC activity since 2000 by Fuks et al. ${ }^{32}$ In this experiment, after HDAC6 was inhibited, DNMT1 was also inhibited. The mechanism of interactive effect was still unknown. However, it could be certain that the combined use of HDACi and DMNTi had a synergistic effect. ${ }^{33,34}$ Since HDACi and DMNTi have been shown to be able to affectthe immune system, the combination of epigenetic drugs and immunosuppressants is considered a promising cancer treatment. ${ }^{35,36}$ At present, for the treatment of acute myeloid leukemia (AML), the combined use of HDACi and DMNTi has entered the stage of clinical trials. ${ }^{37,38}$ For inflammatory diseases, DNMTi 5-Aza 2-deoxycytidine combined with HDACi TSA could reduce acute lung injury caused by endotoxemia. ${ }^{39}$ Therefore, this experiment provides a basis for future use of HDACi in combination with DMNTi to treat liver inflammatory diseases.

In conclusion, ACY-1215 could inhibit the activation of M1 macrophages by improving the glycolytic pathway to alleviate ALF. The mechanism may involve regulating the DNMT1 and DDX3X/NLRP3 pathway. However, the modification mechanisms of DDX3X, MDH1, and IDH1 need to be further studied. This study provided a 
theoretical basis for the clinical application of ACY-1215 in the treatment of ALF in the future.

\section{Data Sharing Statement}

All figures adopted to support the findings of the present study are included in the article.

\section{Acknowledgment}

This work was supported by the Natural Science Foundation of China (81870413, 82070609).

\section{Author Contributions}

All authors contributed to data analysis, drafting, or revising the article, have agreed on the journal to which the article will be submitted, gave final approval of the version to be published, and agree to be accountable for all aspects of the work. Yao Wang and Xun Li contributed equally to this article.

\section{Disclosure}

The authors declare that they have no conflicts of interest for this work.

\section{References}

1. Bernal W, Wendon J. Acute Liver Failure. N Engl J Med. 2013;369 (26):2525-2534.

2. McPhail MJ, Kriese S, Heneghan MA. Current management of acute liver failure. Curr Opin Gastroenterol. 2015;31(3):209-214. doi:10. 1097/MOG.0000000000000174

3. Takaya H, Yoshiji H, Kawaratani H, et al. Decreased activity of plasma ADAMTS13 are related to enhanced cytokinemia and endotoxemia in patients with acute liver failure. Biomed Rep. 2017;7 (3):277-285. doi:10.3892/br.2017.945

4. Wang LK, Wang LW, Li X, et al. Ethyl pyruvate prevents inflammatory factors release and decreases intestinal permeability in rats with D-galactosamine-induced acute liver failure. Hepatobiliary Pancreat Dis Int. 2013;12(2):180-188. doi:10.1016/s1499-3872(13)60029-6

5. Woodhouse CA, Patel VC, Singanayagam A, et al. Review article: the gut microbiome as a therapeutic target in the pathogenesis and treatment of chronic liver disease. Aliment Pharmacol Ther. 2018;47 (2):192-202. doi:10.1111/apt.14397

6. Tomar S, Zumbrun EE, Nagarkatti M, et al. Protective role of cannabinoid receptor 2 activation in galactosamine/lipopolysaccharide-induced acute liver failure through regulation of macrophage polarization and microRNAs. J Pharmacol Exp Ther. 2015;353(2):369-379.

7. van der Heide D, Weiskirchen R, Bansal R. Therapeutic Targeting of Hepatic Macrophages for the Treatment of Liver Diseases. Front Immunol. 2019;10:2852. doi:10.3389/fimmu.2019.02852

8. Galvan-Pena S, O'Neill LA. Metabolic reprograming in macrophage polarization. Front Immunol. 2014;5:420. doi:10.3389/fimmu.2014. 00420

9. O'Neill LA, Kishton RJ, Rathmell J. A guide to immunometabolism for immunologists. Nat Rev Immunol. 2016;16(9):553-565.

10. Peserico A, Simone C. Physical and functional HAT/HDAC interplay regulates protein acetylation balance. $J$ Biomed Biotechnol. 2011;2011:371832. doi:10.1155/2011/371832
11. Farooqi AA, Naqvi SK, Perk AA, et al. Natural Agents-Mediated Targeting of Histone Deacetylases. Arch Immunol Ther Exp (Warsz). 2018;66(1):31-44. doi:10.1007/s00005-017-0488-0

12. Chen Q, Wang Y, Jiao FZ, et al. Histone deacetylase 6 inhibitor ACY1215 offers a protective effect through the autophagy pathway in acute liver failure. Life Sci. 2019;238:116976. doi:10.1016/j. lfs.2019.116976

13. Zhang WB, Yang F, Wang Y, et al. Inhibition of HDAC6 attenuates LPS-induced inflammation in macrophages by regulating oxidative stress and suppressing the TLR4-MAPK/NF-kappaB pathways. Biomed Pharmacother. 2019;117:109166. doi:10.1016/j.biopha.20 19.109166

14. Zhang WB, Zhang HY, Jiao FZ, et al. Histone deacetylase 6 inhibitor ACY-1215 protects against experimental acute liver failure by regulating the TLR4-MAPK/NF-kappaB pathway. Biomed Pharmacother. 2018;97:818-824. doi:10.1016/j.biopha.2017.10.103

15. Samir P, Kesavardhana S, Patmore DM, et al. DDX3X acts as a liveor-die checkpoint in stressed cells by regulating NLRP3 inflammasome. Nature. 2019;573(7775):590-594. doi:10.1038/s41586-019$1551-2$

16. Wendon J, Cordoba J, Dhawan, A., et al.; European Association for the Study of the Liver. Electronic address eee, Clinical practice guidelines p. EASL Clinical Practical Guidelines on the management of acute (fulminant) liver failure. J Hepatol. 2017;66(5):1047-1081. doi:10.1016/j.jhep.2016.12.003

17. Zhang WB, Zhang HY, Wang Y, et al. Quantitative proteomic analysis reveals the sites related to acetylation and mechanism of ACY1215 in acute liver failure mice. Front Pharmacol. 2019;10:653. doi:10.3389/fphar.2019.00653

18. Ryan DG, O'Neill LAJ. Krebs cycle rewired for macrophage and dendritic cell effector functions. FEBS Lett. 2017;591(19):29923006. doi:10.1002/1873-3468.12744

19. Wang Y, Zhang H, Chen Q, et al. TNF-alpha/HMGB1 inflammation signalling pathway regulates pyroptosis during liver failure and acute kidney injury. Cell Prolif. 2020;53(6):e12829.

20. Ambade A, Satishchandran A, Gyongyosi B, et al. Adult mouse model of early hepatocellular carcinoma promoted by alcoholic liver disease. World J Gastroenterol. 2016;22(16):4091-4108. doi:10.3748/wjg.v22.i16.4091

21. Wu Z, Han M, Chen T, et al. Acute liver failure: mechanisms of immune-mediated liver injury. Liver Int. 2010;30(6):782-794. doi:10.1111/j.1478-3231.2010.02262.x

22. Acharya C, Bajaj JS. Gut microbiota and complications of liver disease. Gastroenterol Clin North Am. 2017;46(1):155-169. doi:10.1016/j.gtc.2016.09.013

23. Fukui $H$. Role of gut dysbiosis in liver diseases: what have we learned so far? Diseases. 2019;7(4). doi:10.3390/diseases7040058

24. Liu X, Yu L, Hassan W, et al. The duality of Kupffer cell responses in liver metabolic states. Curr Mol Med. 2016;16(9):809-819. doi:10.2174/1566524016666161031143724

25. Mills E, O'Neill LA. Succinate: a metabolic signal in inflammation. Trends Cell Biol. 2014;24(5):313-320. doi:10.1016/j.tcb.2013.11.008

26. Mills EL, Kelly B, Logan A, et al. Succinate dehydrogenase supports metabolic repurposing of mitochondria to drive inflammatory macrophages. Cell. 2016;167(2):457-470 e413. doi:10.1016/j.cell.2016.08.064

27. Mills EL, Kelly B, O'Neill LAJ. Mitochondria are the powerhouses of immunity. Nat Immunol. 2017;18(5):488-498. doi:10.1038/ni.3704

28. Moran-Salvador E, Mann J. Epigenetics and liver fibrosis. Cell Mol Gastroenterol Hepatol. 2017;4(1):125-134. doi:10.1016/j. jcmgh.2017.04.007

29. Wang Y, Wang L, Gong Z. Regulation of acetylation in high mobility group protein B1 cytosol translocation. DNA Cell Biol. 2019;38 (5):491-499. doi:10.1089/dna.2018.4592

30. Camell CD, Sander J, Spadaro O, et al. Inflammasome-driven catecholamine catabolism in macrophages blunts lipolysis during ageing. Nature. 2017;550(7674):119-123. doi:10.1038/nature24022 
31. Chan $\mathrm{CH}$, Chen CM, Lee YW, et al. DNA damage, liver injury, and tumorigenesis: consequences of DDX3X loss. Mol Cancer Res. 2019;17(2):555-566. doi:10.1158/1541-7786.MCR-18-0551

32. Fuks F, Burgers WA, Brehm A, et al. DNA methyltransferase Dnmt1 associates with histone deacetylase activity. Nat Genet. 2000;24 (1):88-91. doi:10.1038/71750

33. Blagitko-Dorfs N, Schlosser P, Greve G, et al. Combination treatment of acute myeloid leukemia cells with DNMT and HDAC inhibitors: predominant synergistic gene downregulation associated with gene body demethylation. Leukemia. 2019;33(4):945-956. doi:10.1038/ s41375-018-0293-8

34. Jones PA, Issa JP, Baylin S. Targeting the cancer epigenome for therapy. Nat Rev Genet. 2016;17(10):630-641. doi:10.1038/nrg. 2016.93

35. Mazzone R, Zwergel C, Mai A, et al. Epi-drugs in combination with immunotherapy: a new avenue to improve anticancer efficacy. Clin Epigenetics. 2017;9:59. doi:10.1186/s13148-017-0358-y
36. Topper MJ, Vaz M, Chiappinelli KB, et al. Epigenetic therapy ties MYC depletion to reversing immune evasion and treating lung cancer. Cell. 2017;171(6):1284-1300 e1221. doi:10.1016/j.cell.2017. 10.022

37. Wouters BJ, Delwel R. Epigenetics and approaches to targeted epigenetic therapy in acute myeloid leukemia. Blood. 2016;127(1):4252. doi:10.1182/blood-2015-07-604512

38. Tan P, Wei A, Mithraprabhu S, et al. Dual epigenetic targeting with panobinostat and azacitidine in acute myeloid leukemia and high-risk myelodysplastic syndrome. Blood Cancer J. 2014;4:e170. doi:10. 1038/bcj.2013.68

39. Samanta S, Zhou Z, Rajasingh S, et al. DNMT and HDAC inhibitors together abrogate endotoxemia mediated macrophage death by STAT3-JMJD3 signaling. Int J Biochem Cell Biol. 2018;102:117127. doi:10.1016/j.biocel.2018.07.002
Journal of Inflammation Research

\section{Publish your work in this journal}

The Journal of Inflammation Research is an international, peerreviewed open-access journal that welcomes laboratory and clinical findings on the molecular basis, cell biology and pharmacology of inflammation including original research, reviews, symposium reports, hypothesis formation and commentaries on: acute/chronic inflammation; mediators of inflammation; cellular processes; molecular

\section{Dovepress}

mechanisms; pharmacology and novel anti-inflammatory drugs; clinical conditions involving inflammation. The manuscript management system is completely online and includes a very quick and fair peerreview system. Visit http://www.dovepress.com/testimonials.php to read real quotes from published authors. 